\title{
Brain mechanisms of reality monitoring
}

Jon S. Simons ${ }^{1}$, Jane R. Garrison ${ }^{1}$, and Marcia K. Johnson ${ }^{2}$

1. Department of Psychology, University of Cambridge, UK

2. Department of Psychology, Yale University, New Haven, CT, USA.

In Press, Trends in Cognitive Sciences.

Correspondence should be addressed to Dr. Jon Simons, Department of Psychology, University of Cambridge, Downing Street, Cambridge CB2 3EB, UK.

E-mail: jss30@cam.ac.uk.

\section{Acknowledgements}

Preparation of this article was supported by a James S. McDonnell Foundation Scholar Award to JSS, and by the University of Cambridge Behavioural and Clinical Neuroscience Institute, funded by a joint award from the Medical Research Council and the Wellcome Trust. 


\begin{abstract}
Reality monitoring processes are necessary for discriminating between internally-generated information and information that originated in the outside world. They help us identify our thoughts, feelings, and imaginations, and distinguish them from events we may have experienced or been told about by someone else. Reality monitoring errors range from confusions between real and imagined experiences that are byproducts of normal cognition, to symptoms of mental illness such as hallucinations. Recent advances support an emerging neurocognitive characterization of reality monitoring that provides insights into its underlying operating principles and neural mechanisms, the differing ways in which impairment may occur in health and disease, and the potential for rehabilitation strategies to be devised that might help those who experience clinically-significant reality monitoring disruption.
\end{abstract}

\title{
KEYWORDS
}

Source memory, recollection, prefrontal cortex, frontal lobe, hallucinations, schizophrenia 


\section{How Do We Know What is Real?}

Thinking back over your life, you can often find yourself mentally transported back in time, re-living a past episode, sometimes in vivid detail. Except - how do you know that you were actually there when the event originally took place? How can you be sure that you are remembering a faithful representation of what happened, as opposed to an event you might have previously imagined, or a story told to you by someone else? In short, how do you determine whether your memories are real?

One prominent theory, the Source Monitoring Framework, proposes that there are decision processes involved in making attributions about the origins of information that comes to mind, including discriminating information that was generated by internal cognitive functions such as thought and imagination from information that was derived from the outside world by perceptual processes [reality monitoring; 1,2]. According to this framework, memories do not contain propositional tags that directly specify their source. Instead, we make attributions about the origin of a mental experience by considering its features in light of assumptions about the characteristics that tend to be associated with various sources. For example, a person might infer that an apparent "memory" rich in visuoperceptual detail is likely to be real ("I can remember what the dentist's office looked like" [3]) whereas one comprising mainly traces of internally-generated thoughts might instead have been imagined ("I could remember I had a very specific reason for making the association"; "I made the decision by knowing what my train of thought was during the exercise” [4]).

Reality monitoring errors tend to involve misattributing internally-generated events as real, for example later misattributing particularly vivid imaginations to perception, or assuming that the absence of memory for cognitive operations indicates that a memory is unlikely to have been self-generated [4], though misattributions in the other direction also occur, such as in cryptomnesia [5]. Similarity between potential sources increases the likelihood of source errors [6]. For example, misattribution errors are more common for auditory than visual stimuli, perhaps because 'inner speech' and real speech tend to be more 
similar than visual imagery and actual visual perceptions and, hence, more vulnerable to confusion [7]. It is important to note that the features that are activated when a "target" memory is cued are determined by processes engaged during encoding of the event (e.g., the quality of feature binding), during the interval between the initial event and the act of "remembering" (e.g., the number of reactivations), and during retrieval (e.g., the cue eliciting the memory). Also, features from other events can be activated at any point, potentially influencing the characteristics of the remembered event [8]. In addition to the phenomenal qualities of mental experiences, reality monitoring may also involve explicit retrieval of supporting or conflicting information, and may be influenced by prior knowledge, beliefs, and motives [9]. Thus, there are multiple factors operating during encoding and retrieval, and in the intervening period, that can produce source misattributions in healthy individuals, and multiple ways that processes can be disrupted in clinical populations.

In the last few years, a number of laboratories around the world have explored the brain mechanisms underlying reality monitoring processes using cognitive neuroscience methods like functional brain imaging of healthy volunteers and studies of neurological, psychiatric, and developmental disorders, as well as of normal aging [10]. The aim has been to understand how the brain supports our capacity to determine the sources of mental experiences, including distinguishing what is real from what we imagined, an ability that is vital for maintaining confidence in our memories, and in understanding ourselves as individuals in the world with a past and a future. In characterising how these processes might be instantiated in the brain, we can better understand the way in which they may break down in disorders like schizophrenia, in which a person's relation to reality can be altered in ways that disrupt their everyday functioning.

\section{Anterior Prefrontal Cortex and Reality Monitoring}

Neuropsychological and neuroimaging studies have identified a network of brain regions involved in the recollection of source information, which include prefrontal, medial temporal, and parietal cortices [10- 
12]. In broad terms, regions of prefrontal cortex are thought to provide cognitive control over the encoding and retrieval of feature representations that are bound together in a spatial frame by the hippocampus and further integrated by parietal cortex into a first-person perspective that supports the subjective experience of remembering $[9,13]$. The role of the prefrontal cortex appears to be particularly critical for source memory, with lesions to the frontal lobes typically causing severe difficulties with the recollection of such contextual details even when old/new item recognition is unimpaired $[14,15]$.

Distinct prefrontal regions may make separable functional contributions to source memory [11], with ventrolateral PFC sub-regions linked with the specification of retrieval cues and the maintenance of retrieved information, and dorsolateral PFC exhibiting activity during post-retrieval monitoring [16-18].

One region that has emerged as playing a key role in reality monitoring is anterior prefrontal cortex, an area right at the front of the brain that, in relative terms, is roughly twice as large in the human brain as in even the great apes [19]. It has lower cellular density and higher dendritic complexity than comparable cortical regions [20], and is thought to be among the last areas to achieve myelination [21], enabling nerve cells to transmit information more rapidly and facilitating more complex cognitive abilities. As such, although the functions performed by this area are not well understood, they have generally been considered likely to be among the "higher" levels of human complex cognition [21-24]. The role played by anterior PFC in memory has been difficult to characterize. A number of neuroimaging experiments published in the early 2000s reported activation in this region during the recollection of source details $[16,18,25,26]$, but this was not consistently observed $[17,27,28]$. An absence of anterior PFC activity could of course always be attributable to insufficient experimental power or to susceptibility in the fMRI signal due to the proximity of anterior PFC to the sinus area. However, another hypothesis is that the discrepancy between studies might have arisen because the kinds of information that participants were being asked to remember differed according to whether it had been generated internally or externally at the time of encoding [29]. 


\section{INSERT FIGURE 1 ABOUT HERE}

In the past decade or so, this hypothesis has been tested by a number of experiments from several laboratories that have manipulated the recollection of internally-generated and externally-derived source details (Figure 1). Although there is some variability in the patterns of activity elicited by such tasks, these studies have consistently identified the medial aspect of anterior PFC in particular as exhibiting differential activity during the retrieval of internal vs. external aspects of context (Figure 2). For example, medial anterior PFC responses distinguish recollection of the encoding task undertaken compared with remembering where on the screen [29-31] or when in time [32,33] stimuli were presented, or remembering their size on the screen $[25,34]$. The same region is involved in remembering whether verbal phrases were previously presented in full on the screen (e.g., "bacon and eggs"), or whether a word was missing which participants had to imagine (e.g., "bacon and ?") in order to complete the phrase themselves [30,35]. Likewise, medial anterior PFC is differentially engaged during recollection of whether a visual object was previously seen or imagined by participants [36], as well as when judging whether oneself or another person performed a particular action with stimuli [37-39]. The sensitivity of this region to reality monitoring distinctions is apparent regardless of whether words, faces, or objects are being remembered $[29,32,34,36]$, suggesting that the effect is independent of stimulus type. Moreover, medial anterior activation has been observed irrespective of whether the 'internal' or 'external' condition is associated with lower recollection accuracy and longer response times or vice versa, or whether such behavioral factors are matched between conditions [29,32], suggesting that an account in terms of differential task difficulty is unlikely to be sufficient. Possible explanations of the observed activity include that medial anterior PFC represents records of cognitive operations involved in self-generation, or is involved in self-referential processing or self-representation; or that the region operates as a gateway, biasing attention between self-generated and externally-generated information, whether that information is experienced currently or in the past $[21,40]$. 


\section{INSERT FIGURE 2 ABOUT HERE}

Not everybody exhibits perfect reality monitoring performance, of course. Performance varies considerably even in apparently healthy individuals, with some people reliably able to distinguish internally- from externally-generated stimuli and others performing closer to chance levels [41]. Consistent with the functional neuroimaging evidence discussed above, recent research points to these individual differences as having a specific brain structural basis in the medial PFC. One structural landmark in this region of the brain that exhibits considerable morphological variability within the general population is the paracingulate sulcus (PCS), which lies dorsal and parallel to the cingulate sulcus (Figure 3). The PCS, which is prominent in roughly half of the normal population, is a tertiary sulcus, one of the last structural folds to develop before birth and, due to a combination of genetic and environmental influences, varies considerably in size between individuals [42]. This brain structure variation appears to be linked to reality monitoring ability: healthy, apparently cognitively intact, adults whose MRI scans indicate bilateral absence of the PCS are significantly less accurate in reality memory decisions than people with a prominent PCS on at least one side of the brain [41]. PCS reductions are typically associated with increased gray matter volume in surrounding anterior cingulate cortex [43] and, consistent with this observation, voxel-based analyses reveal that reality monitoring performance correlates negatively with medial PFC volume [41]. Reduced sulcal folding and increased surrounding cortical volume may reflect weakened local and long-range connectivity, according to theories of morphogenesis $[44,45]$. These findings thus suggest an explanation for individual differences in reality monitoring ability as reflecting, in part, variations in connectivity between the medial PFC and other cortical regions involved in processing the sensory-perceptual and other features that constitute our memories.

INSERT FIGURE 3 ABOUT HERE 


\section{Reality Monitoring in Schizophrenia}

One of the applications of the work seeking to characterise the brain mechanisms of reality monitoring has been to inform understanding of the cognitive dysfunction seen in clinical conditions, such as schizophrenia. Although schizophrenia can vary in its presentation, among the positive symptoms often observed are hallucinations, such as hearing voices when none are present. For example, a person with schizophrenia might imagine a voice conveying a specific message, and misattribute that voice as real, coming from another person. Hallucinations also occur in other psychiatric conditions including bipolar disorder, major depressive disorder, borderline or schizotypal personality disorder, post-traumatic stress disorder, and dissociative identity disorder [46-48], but affect between 60 and $80 \%$ of patients with a schizophrenia diagnosis $[49,50]$. Auditory and visual hallucinations are the most common forms, with a prevalence of around $59 \%$ and $27 \%$ in schizophrenia, respectively [51], but olfactory, tactile, somatic, and gustatory hallucinations have also been reported [52-54]. Activity associated with hallucinations is often observed in sensory processing areas, such as the superior temporal gyrus during auditory hallucinations and extrastriate cortex during visual hallucinations [55], suggesting separable modality-specific impairments contribute to different types of hallucinations across patients. One possibility is that hallucinations primarily reflect unusually vivid internally-generated experiences in one or more of these modality-specific representational areas, experiences that are so vivid that they seem to be external

events. Another (not necessarily mutually exclusive) possibility is that hallucinations may in part result from a more central difficulty discriminating between perceived and imagined information, perhaps because self-generated events have attenuated or missing records of the cognitive or motor operations that produced them [56].

Despite the interpretational complexities inherent in studying such a heterogeneous syndrome as schizophrenia, a number of predictions have been tested that arise from the hypothesis linking disrupted 
reality monitoring with hallucinations, and the assumption that some common processes are involved in distinguishing reality from imagination both retrospectively and in real time. One prediction is that individuals with schizophrenia should be impaired on the kinds of seen vs. imagined memory tasks that have been shown to elicit anterior PFC activity in healthy volunteers. Of 20 studies of reality monitoring in schizophrenia surveyed for this article (see Table 1), involving a total of 700 patients and 505 controls, 19 studies reported impaired reality monitoring in the patients, including four studies in which the deficit was found to be specific to reality monitoring, with item recognition memory being preserved [57-60]. A second prediction, if hallucinations result from misattributing imagined stimuli as real, is that an externalisation bias should be observed in schizophrenia during reality monitoring performance, with more errors on self-generated than externally-generated trials, and that this bias should be greater in patients with schizophrenia who experience hallucinations compared to those who do not. Of the four studies reporting a specific reality monitoring impairment in schizophrenia, three noted that the deficit only (or disproportionately) involved the misattribution of internally-generated stimuli as having been externally-generated [58-60]. Furthermore, all five studies that compared reality monitoring in patients with and without hallucinations, and measured externalisation bias, observed a greater likelihood of misattributing internally-generated stimuli as real in the patients who experienced hallucinations [57,6164] (see also $[65,66]$ for previous reviews highlighting such associations).

\section{INSERT TABLE 1 ABOUT HERE}

A third prediction is that the anterior PFC region activated in healthy volunteers during reality monitoring performance should be among the areas that are dysfunctional in patients with schizophrenia. Consistent with this prediction, the anterior PFC region linked with reality monitoring in healthy volunteers overlaps closely [30] with one of the areas that consistently exhibit reduced activity in patients with schizophrenia compared with controls during performance of a range of cognitive tasks [67-71]. Moreover, lower anterior PFC activity in healthy individuals during reality monitoring correlates with proneness to 
psychosis and schizotypal trait expression [37], an effect also observed in adolescents at risk of developing schizophrenia [72], suggesting its potential as a possible marker in young people of those with heightened likelihood of converting from prodromal to full psychosis [73].

A fourth prediction is that healthy volunteers who exhibit reduced levels of activity in anterior PFC should make more of the externalisation misattribution errors often observed in schizophrenia. Accordingly, analysis of activity in anterior PFC across participants during reality monitoring performance has revealed a significant negative correlation with the likelihood of mistakenly endorsing imagined items as having been seen [30]. A fifth prediction is that patients with schizophrenia should exhibit disproportionately reduced activity in anterior PFC during performance of reality monitoring tasks, an effect that has been reported in two studies to date $[60,74]$, and which may be partly ameliorated by cognitive training interventions [74] (though this latter possibility requires replication in larger samples). A sixth prediction, if hallucinations in schizophrenia are attributable to overstimulation of sensory processing areas and reality monitoring dysfunction, is that altered functional connectivity should be observed between posterior sensory cortices and anterior PFC. Accordingly, several studies have reported impaired functional integration between superior temporal cortex and medial regions of anterior PFC associated with the misattribution by patients with schizophrenia of their own speech as that of somebody else [75,76]. Finally, based on the specific structural basis for reality monitoring identified in the PCS [41], hallucinations in schizophrenia should be associated with differences in PCS morphology, among other areas. Providing support for this prediction, a quantitative measure of PCS length has been shown to be capable of distinguishing not only patients with schizophrenia from healthy controls [77], but also patients who have experienced hallucinations from patients with no history of such symptoms (Figure 3) [78]. The PCS reduction in patients with hallucinations appears to be evident irrespective of the sensory modality in which they were experienced (e.g., auditory, visual, tactile, olfactory), consistent 
with the observation described above that healthy volunteers exhibit anterior PFC activity during reality monitoring regardless of the kind of stimuli being remembered $[29,32,34,36]$.

\section{Reality Monitoring and Hallucinations}

These findings provide evidence that reality monitoring impairment may be a key component of the cognitive basis for the hallucinations experienced by many patients with schizophrenia. However, it is not only people with a mental health diagnosis who experience hallucinations. Approximately $1 \%$ of the general population report hearing voices when no speaker is present, but do not seek professional help or receive a clinical diagnosis [79]. Phenomenally, the auditory hallucination experience tends to be similar in clinical and non-clinical voice hearers in terms of loudness, involvement of others' voices, number of voices, and so forth, but can differ in frequency, duration, and content, with patients typically experiencing negative voices whereas non-clinical individuals are sometimes more likely to report neutral or pleasant content to their hallucinations [80-82]. Recent interest has focused on whether there might be a common neurocognitive basis for hallucinations in clinical and non-clinical groups. If reduced reality monitoring ability is part of the explanation for the hallucinations observed in schizophrenia, can it also account for the symptoms experienced by non-clinical voice hearers?

Evidence consistent with a common underlying mechanism comes from one study of reality monitoring in healthy volunteers which found that participants who were prone to experience hallucinations were more likely than other participants to misattribute self-generated words as having been spoken by the experimenter [83]. However, two more recent studies involving healthy individuals with a proneness to hallucinations found no evidence of an impairment in source or reality monitoring, or of an increased externalisation bias, in such non-clinical voice hearers [84,85]. Evidence from neuroimaging suggests common hallucination-related activity in auditory processing areas such as the superior temporal gyrus in clinical and non-clinical groups [86], and a correlation between non-clinical participants' auditory 
hallucination proneness scores and activity in superior temporal gyrus when imagining voices for items they subsequently misidentified as heard [87]. Clinical and non-clinical voice-hearers also have similar aberrant microstructure of the arcuate fasciculus connection between frontal and temporal cortices $[88,89]$, but there is as yet no evidence as to whether hallucinations experienced by non-clinical individuals reflect the same pattern of anterior PFC dysfunction that has been observed in patients with schizophrenia.

It may well be that there is more than one route by which hallucinations might occur in clinical and nonclinical groups. Hallucinations are sometimes experienced spontaneously in healthy individuals during periods of sensory deprivation [50], and auditory hallucinations can follow the onset of deafness [90] and visual hallucinations can occur following visual cortex injury [91]. These observations fit with the proposal that hyper-activation of sensory processing cortices might provide the perceptual content for hallucinatory experiences [92]. Consistent with this proposal is evidence that in healthy individuals during periods of silence there is spontaneous random activity in speech sensitive auditory processing areas within the superior temporal gyrus, along with associated activation in the anterior cingulate region of the medial PFC [93]. Thus, it is possible that a hallucination is initiated by spontaneous random activity in sensory processing areas, such as the superior temporal gyrus in the case of auditory hallucinations, reflecting spontaneous 'inner speech' and/or remembered speech, which may occur with greater intensity or frequency during periods of stress or heightened emotion [81]. In healthy individuals who do not experience hallucinations, such sensory activity may be correctly attributed as internallygenerated by reality monitoring processes supported by anterior medial PFC, and experienced as an imagined voice. In patients with schizophrenia who experience hallucinations, spontaneous sensory hyper-activity may be even more intense, and accompanied by hypo-activation of medial PFC regions (consistent with studies of connectivity $[75,76]$ ) resulting in reality monitoring impairment, leading to a failure to recognise the activity as self-generated and to the experience of a hallucination. In non-clinical 
voice hearers, the spontaneous activity in sensory processing areas may either be of such intensity or be so unusual in character (perhaps due to emotional stress, trauma, or tiredness [81]), that an otherwise intact reality monitoring system fails to recognise the stimuli as generated internally and a sporadic hallucination is experienced. A multi-factor model such as this [see also 92,94] can also explain why certain participant groups, such as older adults [95], people with developmental disorders such as autism [96], and healthy volunteers with bilateral PCS absence [41], may exhibit reductions in reality monitoring performance but do not apparently experience hallucinations. These findings may be less compatible with single factor accounts, such as those involving prediction error [e.g. 97].

\section{Concluding Remarks}

There is still much to discover about the brain mechanisms underlying reality monitoring, and the ways in which they may fail in health and disease, impairing the accuracy of judgments about what is real (see Outstanding Questions box). Reality monitoring processes are fundamental for maintaining an understanding of the self as a distinct, conscious agent interacting with the world, perceiving and interpreting external information relating to events happening around us and generating our own thoughts and imaginations and responses. Generally, people are able to keep the 'internal' and 'external' worlds distinguished sufficiently from one another to function under everyday circumstances but, occasionally, anyone may confuse real experiences with those they might have been told about or imagined or dreamt. Some individuals face a greater difficulty in reality monitoring, perhaps reflecting specific individual differences in the general population or, in more severe cases, dysfunction caused by neurological or psychiatric disease.

The evidence from cognitive neuroscience research to date converges to suggest that the anterior PFC region of the brain is central to reality monitoring ability and disability. It is a key component of brain networks that are engaged when distinguishing internally- and externally-generated information, and its disruption is associated with misattributions of reality, confusing internally-generated information as 
having occurred in the outside world. It appears to play an important, and previously underappreciated, role in the psychotic experiences that characterize disorders like schizophrenia. The processes underlying reality monitoring are, like many higher cognitive functions, complex [9], but progress has been made by laboratories around the world in recent years leading to a greater understanding of the brain regions, especially anterior PFC, that subserve reality monitoring mechanisms. An important next challenge will be to apply this knowledge to develop cognitive training techniques and other rehabilitation interventions aimed at enhancing the ability to distinguish real and imagined experiences in people whose sense of reality may be disturbed. Preliminary evidence suggests that such an approach might have potential $[74,98]$, but researchers are just at the beginning of this road and there is much further work to do before it can be determined whether the potential is real. 


\section{Trends Box}

- Mounting evidence identifies anterior prefrontal cortex as playing a key role in reality monitoring, the ability to distinguish internally- from externally-generated information

- Individual differences in reality monitoring performance in healthy volunteers are associated with variability in functional activity and structural morphology in this region of the brain

- Differences are also seen in clinical conditions such as schizophrenia, in which people sometimes experience disturbed awareness of what is real

- Dysfunction in anterior prefrontal cortex increases vulnerability to misattribute internally-generated information as external in origin, providing a possible neurocognitive basis for psychiatric symptoms such as hallucinations 


\section{Outstanding Questions Box}

Although much has been learned in recent years about the mechanisms of reality monitoring, and the way they may be impaired in neurological and psychiatric disorders, there is much still to be discovered. In particular:

- Which regional and interregional brain mechanisms, and related cognitive processes, might explain the intriguing observations that poor reality monitoring performance in healthy individuals is associated with reduced anterior PFC activity, but also with PCS reductions and greater volume of surrounding cortex?

- What are the common and distinct components of neural activity associated with reality monitoring tasks requiring the distinction between self and other, and internal source monitoring between self-generated activities (e.g., imagined and performed)?

- Do hallucinations experienced by non-clinical individuals without a psychiatric diagnosis reflect a similar pattern of anterior PFC dysfunction and reduced PCS length as observed in patients with schizophrenia? Are similar functional and structural markers also present in prodromal individuals who subsequently progress to a diagnosis of schizophrenia?

- What can explain the subjective differences between hallucinations typically experienced by patients with schizophrenia and by non-clinical individuals with a proneness to psychosis? Why are some people with hallucinations fully aware that their experiences are erroneous perceptions, but others are not? 
- Do the range of findings concerning hallucinations require a multiple factor explanation, or can they be explained as fully by more parsimonious single factor accounts, such as prediction error?

- What are the similarities and differences in dysfunction in the brain mechanisms underlying reality monitoring processes that lead to hallucinations, confabulations, and delusions?

- Can interventions that involve cognitive training, brain stimulation, or neurofeedback, be developed according to theoretical hypotheses about disrupted functions to improve reality monitoring ability and, if so, can they be demonstrated to reduce the incidence of hallucinations in people who experience them? 


\section{REFERENCES}

1 Johnson, M.K. and Raye, C.L. (1981) Reality monitoring. Psychological Review 88, 67-85

2 Johnson, M.K. et al. (1993) Source monitoring. Psychological Bulletin 114, 3-28

3 Johnson, M.K. et al. (1988) Phenomenal characteristics of memories for perceived and imagined autobiographical events. Journal of Experimental Psychology: General 117, 371-376

4 Johnson, M.K. et al. (1981) Cognitive operations and decision bias in reality monitoring. The American Journal of Psychology 94, 37-64

5 Macrae, C.N. et al. (1999) Contexts of cryptomnesia: May the source be with you. Social Cognition 17, 273-297

6 Johnson, M.K. et al. (1988) The consequences for memory of imagining in another person's voice. Mem Cognit 16, 337-342

7 Garrison, J.R. et al. (2017) Monitoring what is real: The effects of modality and action on accuracy and type of reality monitoring error. Cortex $87,108-117$

8 Lyle, K.B. and Johnson, M.K. (2006) Importing perceived features into false memories. Memory 14, 197-213

9 Johnson, M.K. et al. (2012) The cognitive neuroscience of true and false memories. In True and False Recovered Memories 58 (Belli, R. F., ed), pp. 15-52, Springer New York

10 Mitchell, K.J. and Johnson, M.K. (2009) Source monitoring 15 years later: What have we learned from $\mathrm{fMRI}$ about the neural mechanisms of source memory? Psychological Bulletin 135, 638-677

11 Simons, J.S. and Spiers, H.J. (2003) Prefrontal and medial temporal lobe interactions in long-term memory. Nature Reviews Neuroscience 4, 637-648

12 Rugg, M.D. and Vilberg, K.L. (2013) Brain networks underlying episodic memory retrieval. Current Opinion in Neurobiology 23, 255-260

13 Moscovitch, M. et al. (2016) Episodic memory and beyond: The hippocampus and neocortex in transformation. Annual Review of Psychology 67, 105-134

14 Janowsky, J.S. et al. (1989) Source memory impairment in patients with frontal lobe lesions. Neuropsychologia 27, 1043-1056

15 Simons, J.S. et al. (2002) Recollection-based memory in frontotemporal dementia: Implications for theories of long-term memory. Brain 125, 2523-2536

16 Rugg, M.D. et al. (1999) The role of the prefrontal cortex in recognition memory and memory for source: An fMRI study. Neurolmage 10, 520-529

17 Henson, R.N.A. et al. (1999) Right prefrontal cortex and episodic memory retrieval: A functional MRI test of the monitoring hypothesis. Brain 122, 1367-1381

18 Dobbins, I.G. et al. (2002) Executive control during episodic retrieval: Multiple prefrontal processes subserve source memory. Neuron 35, 989-996

19 Semendeferi, K. et al. (2001) Prefrontal cortex in humans and apes: A comparative study of area 10. American Journal of Physical Anthropology 114, 224-241

20 Jacobs, B. et al. (2001) Regional dendritic and spine variation in human cerebral cortex: A quantitative golgi study. Cereb. Cortex 11, 558-571

21 Burgess, P.W. et al. (2005) The gateway hypothesis of rostral prefrontal cortex (area 10) function. In Measuring the Mind: Speed, Control, and Age (Duncan, J. et al., eds), pp. 217-248, Oxford University Press

22 Koechlin, E. et al. (1999) The role of the anterior prefrontal cortex in human cognition. Nature 399, 148-151

23 Ramnani, N. and Owen, A.M. (2004) Anterior prefrontal cortex: Insights into function from anatomy and neuroimaging. Nature Reviews Neuroscience 5, 184-194 
24 Badre, D. (2008) Cognitive control, hierarchy, and the rostro-caudal organization of the frontal lobes. Trends in Cognitive Sciences 12, 193-200

25 Ranganath, C. et al. (2000) Left anterior prefrontal activation increases with demands to recall specific perceptual information. Journal of Neuroscience 20:RC108, 1-5

26 Kahn, l. et al. (2004) Functional-neuroanatomic correlates of recollection: Implications for models of recognition memory. Journal of Neuroscience 24, 4172-4180

27 Nyberg, L. et al. (1996) General and specific brain regions involved in encoding and retrieval of events: What, where, and when. Proceedings of the National Academy of Sciences USA 93, 1128011285

28 Suzuki, M. et al. (2002) Neural basis of temporal context memory: A functional MRI study. Neurolmage 17, 1790-1796

29 Simons, J.S. et al. (2005) Anterior prefrontal cortex and the recollection of contextual information. Neuropsychologia 43, 1774-1783

30 Simons, J.S. et al. (2006) Discriminating imagined from perceived information engages brain areas implicated in schizophrenia. Neurolmage 32, 696-703

31 Gilbert, S.J. et al. (2010) The scale of functional specialization within human prefrontal cortex. Journal of Neuroscience 30, 1233-1237

32 Simons, J.S. et al. (2005) Distinct roles for lateral and medial anterior prefrontal cortex in contextual recollection. Journal of Neurophysiology 94, 813-820

33 Turner, M.S. et al. (2008) Distinct roles for lateral and medial rostral prefrontal cortex in source monitoring of perceived and imagined events. Neuropsychologia 46, 1442-1453

34 Dobbins, I.G. and Wagner, A.D. (2005) Domain-general and domain-sensitive prefrontal mechanisms for recollecting events and detecting novelty. Cerebral Cortex 15, 1768-1778

35 Vinogradov, S. et al. (2006) Brain activation patterns during memory of cognitive agency. Neurolmage 31, 896-905

36 Kensinger, E.A. and Schacter, D.L. (2006) Neural processes underlying memory attribution on a reality-monitoring task. Cerebral Cortex 16, 1126-1133

37 Simons, J.S. et al. (2008) Separable forms of reality monitoring supported by anterior prefrontal cortex. Journal of Cognitive Neuroscience 20, 447-457

38 Brandt, V.C. et al. (2014) Did I turn off the gas? Reality monitoring of everyday actions. Cogn Affect Behav Neurosci 14, 209-219

39 Metzak, P.D. et al. (2015) Functional brain networks involved in reality monitoring. Neuropsychologia 75, 50-60

40 Johnson, M.K. (2016) Cognitive Neuroscience: Applied Cognitive Psychology. Journal of Applied Research in Memory and Cognition 5, 110-120

41 Buda, M. et al. (2011) A specific brain structural basis for individual differences in reality monitoring. Journal of Neuroscience 31, 14308-14313

42 Armstrong, E. et al. (1995) The ontogeny of human gyrification. Cereb. Cortex 5, 56-63

43 Fornito, A. et al. (2008) Variability of the paracingulate sulcus and morphometry of the medial frontal cortex: Associations with cortical thickness, surface area, volume, and sulcal depth. Human Brain Mapping 29, 222-236

44 Van Essen, D.C. (1997) A tension-based theory of morphogenesis and compact wiring in the central nervous system. Nature 385, 313-318

45 Ronan, L. et al. (2014) Differential tangential expansion as a mechanism for cortical gyrification. Cereb. Cortex 24, 2219-2228

46 Siegel, R.K. (1984) Hostage hallucinations. Visual imagery induced by isolation and life-threatening stress. J. Nerv. Ment. Dis. 172, 264-272 
47 Ross, C.A. et al. (1990) Schneiderian symptoms in multiple personality disorder and schizophrenia. Comprehensive Psychiatry 31, 111-118

48 Skaf, C.R. et al. (2002) Psychotic symptoms in major depressive disorder are associated with reduced regional cerebral blood flow in the subgenual anterior cingulate cortex: a voxel-based single photon emission computed tomography (SPECT) study. Journal of Affective Disorders 68, 295305

49 Wing, J.K. et al. (1974) Measurement and classification of psychiatric symptoms, Cambridge University Press.

50 Slade, P.D. and Bentall, R.P. (1988) Sensory deception: A scientific analysis of hallucination, Johns Hopkins University Press.

51 Waters, F. et al. (2014) Visual hallucinations in the psychosis spectrum and comparative information from neurodegenerative disorders and eye disease. Schizophrenia Bulletin 40, S233-S245

52 Mueser, K.T. et al. (1990) Hallucinations in schizophrenia. Acta Psychiatrica Scandinavica 82, 26-29

53 Kopala, L.C. et al. (1994) Olfactory hallucinations and olfactory identification ability in patients with schizophrenia and other psychiatric disorders. Schizophrenia Research 12, 205-211

54 Shergill, S.S. et al. (2001) Modality specific neural correlates of auditory and somatic hallucinations. Journal of Neurology, Neurosurgery \& Psychiatry 71, 688-690

55 Zmigrod, L. et al. (2016) The neural mechanisms of hallucinations: A quantitative meta-analysis of neuroimaging studies. Neuroscience \& Biobehavioral Reviews 69, 113-123

56 Frith, C.D. and Done, D.J. (1989) Experiences of alien control in schizophrenia reflect a disorder in the central monitoring of action. Psychological Medicine 19, 359-363

57 Seal, M.L. et al. (1997) Deficits in source monitoring in subjects with auditory hallucinations may be due to differences in verbal intelligence and verbal memory. Cognitive Neuropsychiatry 2, 273-290

58 Stephane, M. et al. (2010) Evaluation of speech misattribution bias in schizophrenia. Psychological Medicine 40, 741-748

59 Vinogradov, S. et al. (1997) Clinical and neurocognitive aspects of source monitoring errors in schizophrenia. American Journal of Psychiatry 154, 1530-1537

60 Vinogradov, S. et al. (2008) Deficit in a neural correlate of reality monitoring in schizophrenia patients. Cerebral Cortex 18, 2532-2539

61 Woodward, T.S. et al. (2007) Source monitoring biases and auditory hallucinations. Cognitive Neuropsychiatry 12, 477-494

62 Bentall, R.P. et al. (1991) Reality monitoring and psychotic hallucinations. British Journal of Clinical Psychology 30, 213-222

63 Brébion, G. et al. (2000) Positive symptomatology and source-monitoring failure in schizophrenia-an analysis of symptom-specific effects. Psychiatry Res 95, 119-131

64 Brunelin, J. et al. (2007) Impaired verbal source monitoring in schizophrenia: An intermediate trait vulnerability marker? Schizophrenia Research 89, 287-292

65 Waters, F. et al. (2012) Self-recognition Deficits in Schizophrenia Patients With Auditory Hallucinations: A Meta-analysis of the Literature. Schizophr Bull 38, 741-750

66 Brookwell, M.L. et al. (2013) Externalizing biases and hallucinations in source-monitoring, selfmonitoring and signal detection studies: a meta-analytic review. Psychological Medicine 43, 24652475

67 Andreasen, N.C. et al. (1996) Schizophrenia and cognitive dysmetria: A positron-emission tomography study of dysfunctional prefrontal-thalamic-cerebellar circuitry. Proceedings of the National Academy of Sciences USA 93, 9985-9990

68 Callicott, J.H. et al. (2003) Abnormal fMRI response of the dorsolateral prefrontal cortex in cognitively intact siblings of patients with schizophrenia. American Journal of Psychiatry 160, 709719 
69 Whalley, H.C. et al. (2004) fMRI correlates of state and trait effects in subjects at genetically enhanced risk of schizophrenia. Brain 127, 478-490

70 Fox, M.D. et al. (2005) The BOLD onset transient: Identification of novel functional differences in schizophrenia. Neurolmage 25, 771-782

71 MacDonald, A.W. et al. (2005) Specificity of prefrontal dysfunction and context processing deficits to schizophrenia in never-medicated patients with first-episode psychosis. American Journal of Psychiatry 162, 475-484

72 Lagioia, A.-L. et al. (2011) Neural correlates of reality monitoring during adolescence. Neurolmage $55,1393-1400$

73 Cannon, T.D. (2015) How schizophrenia develops: Cognitive and brain mechanisms underlying onset of psychosis. Trends in Cognitive Sciences 19, 744-756

74 Subramaniam, K. et al. (2012) Computerized cognitive training restores neural activity within the reality monitoring network in schizophrenia. Neuron 73, 842-853

75 Mechelli, A. et al. (2007) Misattribution of speech and impaired connectivity in patients with auditory verbal hallucinations. Hum. Brain Mapp. 28, 1213-1222

76 Wang, L. et al. (2011) Aberrant connectivity during self-other source monitoring in schizophrenia. Schizophrenia Research 125, 136-142

77 Fornito, A. et al. (2006) Morphology of the paracingulate sulcus and executive cognition in schizophrenia. Schizophrenia Research 88, 192-197

78 Garrison, J.R. et al. (2015) Paracingulate sulcus morphology is associated with hallucinations in the human brain. Nat Commun 6, 8956

79 Johns, L.C. et al. (2004) Prevalence and correlates of self-reported psychotic symptoms in the British population. Br J Psychiatry 185, 298-305

80 Daalman, K. et al. (2011) The same or different? A phenomenological comparison of auditory verbal hallucinations in healthy and psychotic individuals. The Journal of clinical psychiatry 72, 320-325

81 Johns, L.C. et al. (2014) Auditory Verbal Hallucinations in Persons With and Without a Need for Care. Schizophrenia Bulletin 40, S255-S264

82 Woods, A. et al. (2015) Experiences of hearing voices: analysis of a novel phenomenological survey. The Lancet Psychiatry 2, 323-331

83 Larøi, F. et al. (2004) The effects of emotional salience, cognitive effort and meta-cognitive beliefs on a reality monitoring task in hallucination-prone subjects. British Journal of Clinical Psychology 43, 221-233

84 McKague, M. et al. (2012) Source monitoring and proneness to auditory-verbal hallucinations: A signal detection analysis. Cognitive Neuropsychiatry 17, 544-562

85 Garrison, J.R. et al. (2017) Testing continuum models of psychosis: No reduction in source monitoring ability in healthy individuals prone to auditory hallucinations. Cortex DOI: 10.1016/j.cortex.2016.11.011

86 Allen, P. et al. (2012) Neuroimaging Auditory Hallucinations in Schizophrenia: From Neuroanatomy to Neurochemistry and Beyond. Schizophr Bull 38, 695-703

87 Sugimori, E. et al. (2014) Brain Mechanisms Underlying Reality Monitoring for Heard and Imagined Words. Psychological Science 25, 403-413

88 de Weijer, A.D. et al. (2013) Aberrations in the arcuate fasciculus are associated with auditory verbal hallucinations in psychotic and in non-psychotic individuals. Hum Brain Mapp 34, 626-634

89 McCarthy-Jones, S. et al. (2015) Reduced integrity of the left arcuate fasciculus is specifically associated with auditory verbal hallucinations in schizophrenia. Schizophrenia Research 162, 1-6

90 Thewissen, V. et al. (2005) Hearing impairment and psychosis revisited. Schizophr. Res. 76, 99-103

91 Kölmel, H.W. (1985) Complex visual hallucinations in the hemianopic field. J. Neurol. Neurosurg. Psychiatr. 48, 29-38 
92 Allen, P. et al. (2008) The hallucinating brain: A review of structural and functional neuroimaging studies of hallucinations. Neuroscience \& Biobehavioral Reviews 32, 175-191

93 Hunter, M.D. et al. (2006) Neural activity in speech-sensitive auditory cortex during silence. PNAS $103,189-194$

94 Jones, S.R. and Fernyhough, C. (2009) Rumination, reflection, intrusive thoughts, and hallucinationproneness: Towards a new model. Behaviour Research and Therapy 47, 54-59

95 Henkel, L.A. et al. (1998) Aging and source monitoring: Cognitive processes and neuropsychological correlates. Journal of Experimental Psychology: General 127, 251-268

96 Cooper, R.A. et al. (2016) Reality monitoring and metamemory in adults with autism spectrum conditions. Journal of Autism and Developmental Disorders 46, 2186-2198

97 Fletcher, P.C. and Frith, C.D. (2008) Perceiving is believing: a Bayesian approach to explaining the positive symptoms of schizophrenia. Nature Reviews Neuroscience 10, 48-58

98 Mammarella, N. et al. (2017) Self-generation and positivity effects following transcranial random noise stimulation in medial prefrontal cortex: a reality monitoring task in older adults. Cortex DOI: 10.1016/j.cortex.2016.11.005 


\section{Figure Legends}

\section{Figure 1}

Three examples illustrating the variety of reality monitoring tasks and anterior prefrontal cortex activity elicited. Top panel - memory for encoding task vs. stimulus size [34] (fMRI image courtesy of Ian G Dobbins, PhD). Middle panel - remembering whether words were seen or imagined [35] (fMRI image reprinted with permission from Elsevier). Bottom panel - distinguishing word pairs read aloud by the participant herself vs. the experimenter [37] (fMRI image reprinted with permission from MIT Press).

\section{Figure 2}

Locations of medial anterior prefrontal cortex activity reported by $12 \mathrm{fMRI}$ studies of reality monitoring in healthy volunteers.

\section{Figure 3}

Paracingulate sulcus (PCS) and reality monitoring. Top panel - The PCS (shown in red), located in the medial PFC dorsal to the cingulate sulcus (CS), differs in length considerably between people (reprinted with permission from Nature Publishing Group). Bottom left panel - Reduced reality monitoring performance in healthy volunteers in whom PCS absent in both hemispheres [41]. Bottom right panelPCS length differentiates hallucination status in patients with schizophrenia, as well as distinguishing patients with schizophrenia from healthy control participants [78] (reprinted with permission for Nature Publishing Group). 
Table 1: Studies of Reality Monitoring in Schizophrenia

\begin{tabular}{|c|c|c|c|c|c|c|}
\hline \multirow[t]{2}{*}{ Study } & \multirow[t]{2}{*}{ Task } & \multicolumn{2}{|c|}{ Subjects } & \multicolumn{2}{|c|}{ Impairment in } & \multirow{2}{*}{$\begin{array}{c}\text { Increased } \\
\text { Externalisation } \\
\text { Bias } \\
\end{array}$} \\
\hline & & Patients (N) & Controls (N) & $\begin{array}{c}\text { Recognition } \\
\text { Memory }\end{array}$ & $\begin{array}{c}\text { Reality } \\
\text { Monitoring }\end{array}$ & \\
\hline \multicolumn{7}{|c|}{ Patients with schizophrenia versus healthy contro/s } \\
\hline Anselmetti et al. (2007) & Word-stem completion & 45 & 54 & Yes & Yes & Yes \\
\hline Bentall et al. (1991) & Word-stem completion & 22 & 22 & - & Yes & No \\
\hline Brebion et al. (2000) & Semantic association & 40 & 40 & - & Yes & - \\
\hline Brunelin et al. (2007) & Word recognition & 15 & 15 & - & Yes & - \\
\hline Brunelin et al. (2008) & Word recognition & 30 & 24 & - & Yes & - \\
\hline Fisher et al. (2008) & Sentence completion & 91 & 30 & Yes & Yes & Yes \\
\hline Franck et al. (2000) & Word recognition & 17 & 17 & - & Yes & Yes \\
\hline Harvey et al. (1988) & Word recognition & 26 & 25 & - & Yes & - \\
\hline Keefe et al. (2002) & Word-stem completion & 29 & 19 & Yes & Yes & Yes \\
\hline Moritz et al. (2003) & Semantic association & 30 & 21 & Yes & Yes & No \\
\hline Moritz et al. (2005) & Semantic association & 30 & 15 & Yes & No & No \\
\hline Nienow \& Docherty (2004) & Sentence completion & 52 & 52 & Yes & Yes & Yes \\
\hline Seal et al. (1997) & Semantic association & 21 & 15 & No & Yes & No \\
\hline Stephane et al. (2010) & Sentence recognition & 39 & 26 & No & Yes & Yes \\
\hline Subramaniam et al. (2012) & Sentence completion & 31 & 16 & - & Yes & Yes \\
\hline Szoke et al. (2009) & Semantic association & 54 & 41 & - & Yes & No \\
\hline Vinogradov et al. (1997) & Sentence completion & 26 & 21 & No & Yes & Yes \\
\hline Vinogradov et al. (2008) & Sentence completion & 8 & 8 & No & Yes & Yes \\
\hline Waters et al. (2004) & Object pairing & 43 & 24 & Yes & Yes & No \\
\hline Woodward et al. (2007) & Sentence completion & 51 & 20 & Yes & Yes & No \\
\hline Total subjects & & 700 & 505 & & & \\
\hline \multirow[t]{2}{*}{ Study } & Task & \multicolumn{2}{|c|}{ Subjects } & \multicolumn{2}{|c|}{ Impairment in } & Increased \\
\hline & & Hall (N) & Non-Hall (N) & $\begin{array}{c}\text { Recognition } \\
\text { Memory }\end{array}$ & $\begin{array}{c}\text { Reality } \\
\text { Monitoring }\end{array}$ & $\begin{array}{c}\text { Externalisation } \\
\text { Bias }\end{array}$ \\
\hline \multicolumn{7}{|c|}{ Patients with schizophrenia: hallucinations vs non-hallucinations } \\
\hline Bentall et al. (1991) & Word-stem completion & 22 & 16 & - & No & Yes \\
\hline Brebion et al. (2000) & Semantic association & 22 & 18 & - & No & Yes \\
\hline Brunelin et al. (2006) & Word recognition & 30 & 31 & No & Yes & Yes \\
\hline Seal et al. (1997) & Semantic association & 10 & 11 & - & No & Yes \\
\hline Waters et al. (2006) & Object pairing & 19 & 24 & No & Yes & - \\
\hline Woodward et al. (2007) & Sentence completion & 16 & 35 & No & No & Yes \\
\hline Total subjects & & 119 & 135 & & & \\
\hline
\end{tabular}


Figure 1

Study Phase

Test Phase
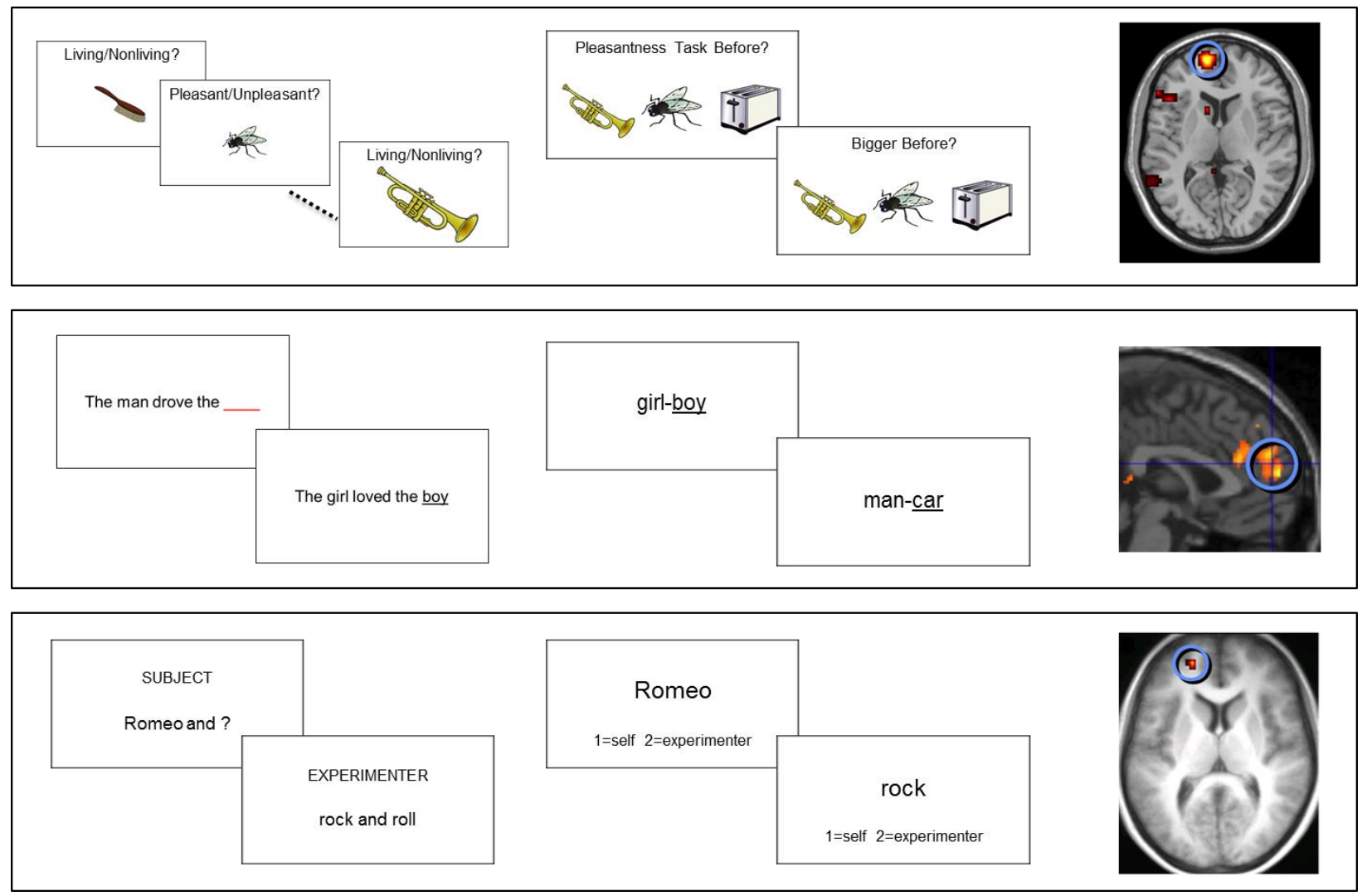
Figure 2
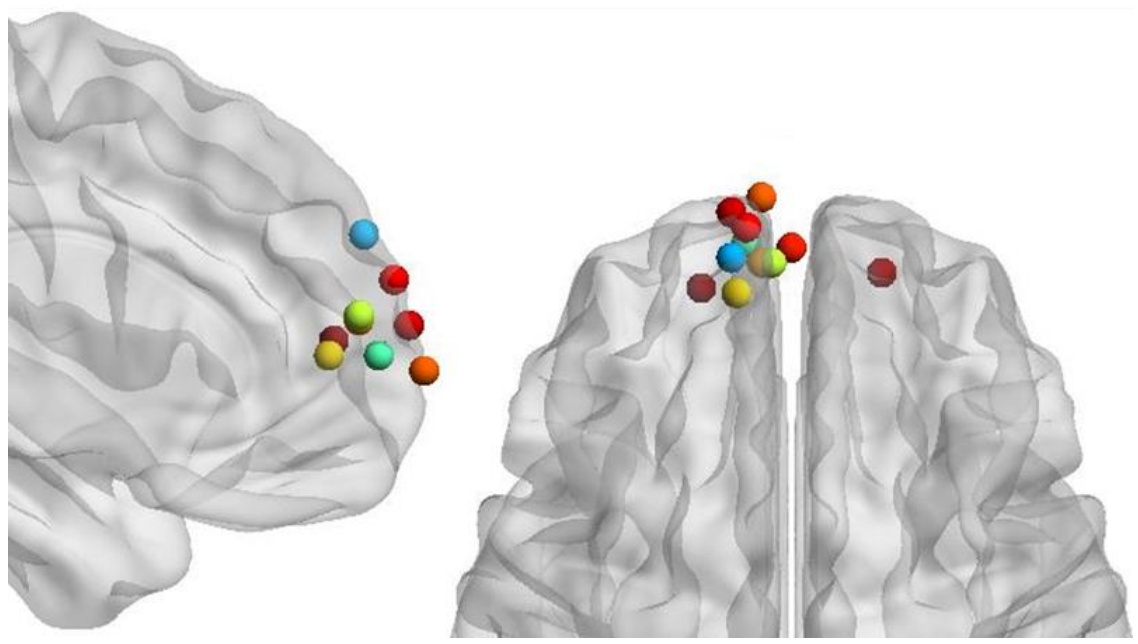
Figure 3
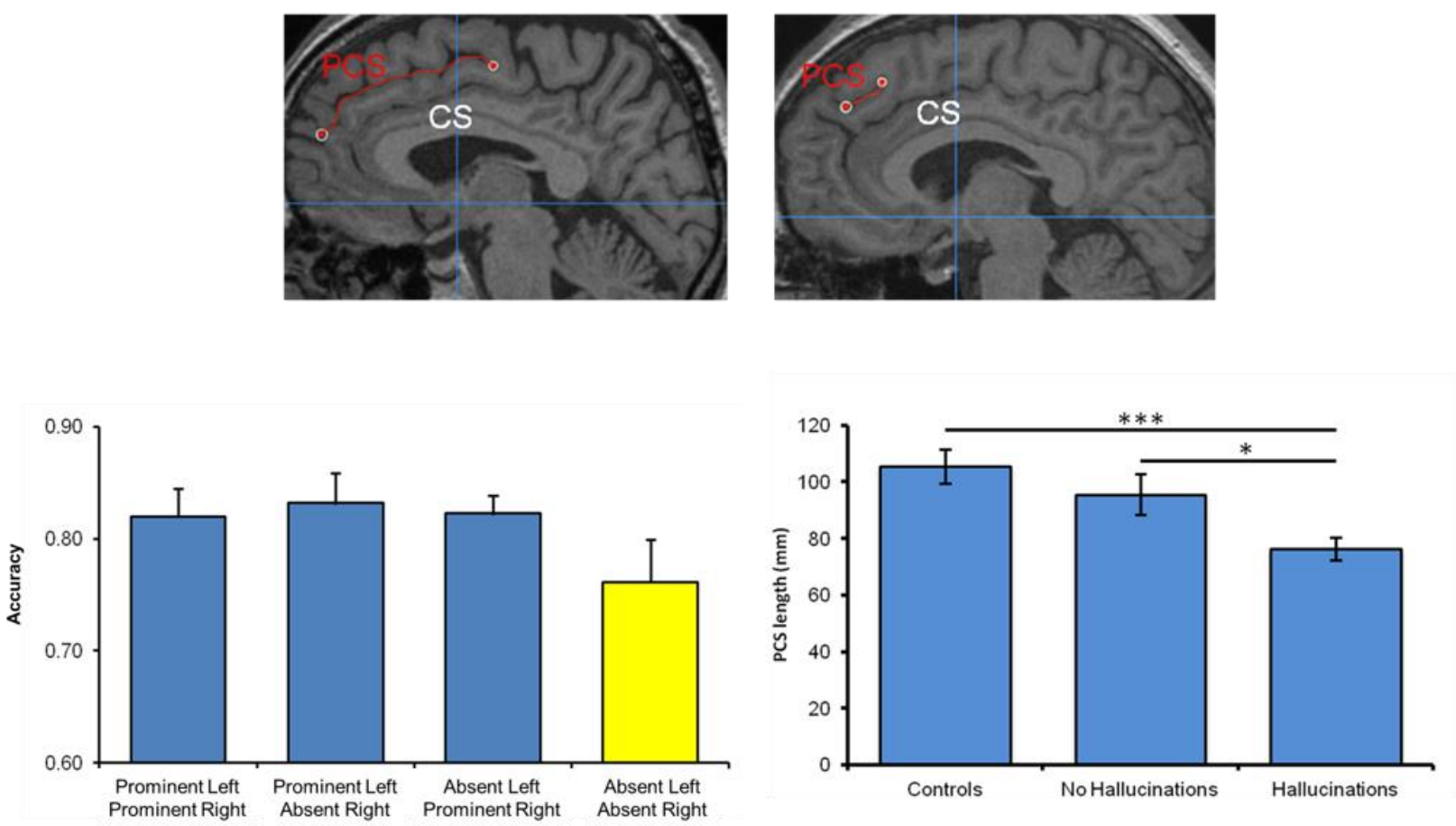